\title{
Oxidative damage to human parametrial ligament fibroblasts induced by mechanical stress
}

\author{
SHASHA HONG, LI HONG, DEBIN WU, BINGSHU LI, CHENG LIU, \\ WENJUN GUO, JIE MIN, MING HU, YANG ZHAO and QING YANG
}

\begin{abstract}
Department of Gynaecology and Obstetrics, Renmin Hospital of Wuhan University, Wuhan, Hubei 430060, P.R. China
\end{abstract}
Received October 15, 2014; Accepted July 3, 2015

DOI: $10.3892 / \mathrm{mmr} .2015 .4115$

\begin{abstract}
The aim of the present study was to explore the underlying mechanisms of the roles of mechanical factors in the pathogenesis of pelvic organ prolapse (POP). The experiments were performed on fibroblasts derived from uterosacral ligaments and cardinal ligaments of patients who received total hysterectomy due to benign disease excluding POP. Fibroblasts were cultured after collagenase digestion and identified by morphological observation and immunocytochemical methods. A four-point bending device was used to subject fibroblasts at passage 4-6 to strains of $0,1,333 \mu(1 \mathrm{~mm}), 2,666 \mu$ $(2 \mathrm{~mm})$ or $5,333 \mu(4 \mathrm{~mm})$ at a frequency of $0.1 \mathrm{~Hz}$ for $4 \mathrm{~h}$. Intracellular reactive oxygen species (ROS) were quantified using the fluorescent probe 2',7'-dichlorodihydrofluorescein diacetate. Changes in the mitochondrial membrane potential were verified using the fluorescent dye JC-1, and apoptosis was detected using Annexin V/propidium iodide staining and flow cytometric analysis. Mechanical strain changed the morphology and adherence ability of parametrial ligament fibroblasts. Furthermore, the production of ROS was significantly increased and the mitochondrial membrane potential obviously declined with the enhancement of mechanical stress loading. In addition, the apoptotic rate of fibroblasts subjected to high mechanical strain was significantly increased compared with that in fibroblast under low-intensity strain. In conclusion, the present study showed that mechanical strain enhanced intracellular ROS levels, decreased the mitochondrial membrane potential and increased the apoptotic rate in human parametrial ligament fibroblasts, which may contribute to POP.
\end{abstract}

Correspondence to: Dr Li Hong, Department of Gynaecology and Obstetrics, Renmin Hospital of Wuhan University, 238 Jiefang Road, Wuhan, Hubei 430060, P.R. China

E-mail: drhongli7777@gmail.com

Key words: mechanical stress, fibroblast, oxidative stress, apoptosis, pelvic organ prolapse

\section{Introduction}

Pelvic organ prolapse (POP) is a common disease whose treatment is costly and which affects millions of women worldwide. Research shows that $\sim 30 \%$ of elderly women suffer from prolapse to a certain degree (1). Regarding the consequences of POP, it can negatively impair the quality of the patients' life and cause serious inconveniences to the patients and their families.

Pelvic organs are supported by levator ani muscles and connective tissue (endopelvic fascia and ligaments) attached to the pelvic organs. Therefore, the disruption or dysfunction of these supportive components may lead to a loss of support and eventually to POP (2). Gestation and delivery can increase intra-abdominal pressure and damage the muscles and fascia in the pelvic floor, which is a well-known risk factor for POP. However, the underlying mechanisms of the effects of pelvic mechanical forces on POP has remained elusive.

Cells of the human body are equipped with an anti-oxidant system. Upon excessive production of reactive oxygen species (ROS) and the presence of excess levels of metabolites, the redox balance is disturbed, which activates anti-oxidant systems and triggers an oxidative stress reaction $(3,4)$.

It has been suggested that mechanical forces can activate oxidative stress signaling pathways. This process is caused by a redox imbalance, which further leads to vascular remodeling (5). Cyclic mechanical forces were shown to significantly increase the levels of intracellular ROS in myoblasts in a dose-dependent manner (6).

Furthermore, a close correlation between POP and oxidative stress in the human body has been evidenced. The content of isoprostanes, which are oxidative stress-associated factors, was significantly increased in the cardinal ligament of the uterus and in the urine of patients with uterine prolapse (7). The expression of the anti-oxidant-associated gene DSCR-1 decreased by 5.1 times in patients with POP (8). In skin fibroblasts, oxidative stress disturbs collagen synthesis and gene expression (9). Therefore, the present study hypothesized that the oxidative stress pathway is involved in the development process of POP.

Mitochondria, as the center of cellular energy metabolism and redox reactions, are the main cellular site of ROS generation. At the same time mitochondria are important target organelles of oxidative damage (10). Oxidative stress changes the mitochondrial membrane permeability, decreases 
the mitochondrial membrane potential $(\Delta \Psi \mathrm{m})$ and suppresses the functions of the mitochondria. Consequently, caspase-3 is activated by pro-apoptotic molecules such as cytochrome $C$ from mitochondria, which eventually leads to cell apoptosis (10). A previous study found that the apoptotic rate was significantly increased in the supportive tissue of the pelvic floor of POP patients (11). Caspase-3 and -9 expression was significantly elevated in the sacral ligament of patients with POP, which confirmed the involvement of mitochondrial apoptosis in the pathogenesis of POP (10).

The aim of the present study was to determine the influence of mechanical strain on human parametrial ligament fibroblasts (HPLFs), as well as to investigate the underlying mechanisms of the effects. The levels of ROS, changes in the mitochondrial membrane potential and the apoptotic rate were determined in order to explore the possible mechanisms of mechanical factors in the pathogenesis of POP.

\section{Materials and methods}

Ethics statement. The present study was performed on human subjects and was approved by the Ethics Committee of Renmin Hospital of Wuhan University (Wuhan, China). Written informed consent was obtained from all patients prior to participation in the study.

Participants. A total of 10 patients who underwent hysterectomy surgery for reasons excluding the presence of malignant tumors and POP, were enrolled in the present study. None of the recruited women had any connective tissue diseases, pathologically confirmed endometriosis or estrogen-associated ovarian tumors. Furthermore, the patients were free from any complications that may lead to oxidative stress-associated diseases, including coronary heart disease, diabetes and hyperlipidemia. Patients who received surgery in the uterosacral ligamental site or had a history of estrogen application within the past three months were excluded from the present study.

Primary cell culture. The present study adopted a modified enzyme digestion method (12) for the establishment of primary cell cultures. Tissue specimens $\left(0.5 \times 0.5 \times 0.2 \mathrm{~cm}^{3}\right)$ were obtained from part of the parametrial ligament during surgery (including sacral ligament and cardinal ligaments). The samples were washed with phosphate-buffered saline (PBS) containing $100 \mathrm{KU} / \mathrm{ml}$ penicillin $\mathrm{G}$ and $100 \mathrm{mg} / \mathrm{ml}$ streptomycin (Jenom, Hangzhou, China), and then minced into small pieces. The tissues were digested with $1 \%$ collagenase-I (Invitrogen Life Technologies, Inc., Carlsbad, CA, USA) for $3 \mathrm{~h}$ at $37^{\circ} \mathrm{C}$ in $5 \% \mathrm{CO}_{2}$, followed by further digestion with $0.25 \%$ trypsin (Sigma-Aldrich, St. Louis, MO, USA) for $5 \mathrm{~min} .2 \mathrm{ml}$ fetal bovine serum (FBS; Gibco-BRL, Invitrogen Life Technologies) was used to stop the digestion. Dulbecco's modified Eagle's medium (DMEM; Jenom, Hangzhou, China) containing 15\% FBS was then slowly added to the culture flask. The medium was replaced every two days and the primary HPLF cell cultures were grown to confluence for passage. The HPLFs were used at passage 4-8 for the subsequent experiments.

Immunocytochemical staining. Cells in the logarithmic growth phase were digested with $0.25 \%$ trypsin, re-suspended and seeded into a six-well plate containing a pre-placed coverslip, followed by incubation for two days at a cell density of $10^{5}$ cells $/ \mathrm{ml}$. After cells were $\sim 70 \%$ confluent, the coverslips were removed. Cells were washed twice in PBS and immersed in $4 \%$ paraformaldehyde for $30 \mathrm{~min}$ for fixation. Cells then were washed three times in PBS, immersed in $3 \%$ $\mathrm{H}_{2} \mathrm{O}_{2}$ and then incubated in the dark. After the endogenous peroxidase activity was exhausted, cells were washed three times in PBS and then incubated with mouse monoclonal vimentin (cat. no. sc-6260; Santa Cruz Biotechnology, Inc., Dallas, TX, USA; $200 \mu \mathrm{g} / \mathrm{ml}$ ), and mouse monoclonal cytokeratin-19 (cat. no. sc-6278; Santa Cruz Biotechnology, Inc.; $200 \mu \mathrm{g} / \mathrm{ml}$ ) antibodies at $4^{\circ} \mathrm{C}$ overnight followed by three washes in PBS. Subsequently, the cells were incubated with secondary antibodies (k5007; Dako, Glostrup, Denmark) at room temperature in the dark for $1 \mathrm{~h}$ followed by three washes in PBS. The immunohistochemical stain was evaluated using an upright microscope (BX51; Olympus, Tokyo, Japan).

Compression of human parametrial ligament fibroblasts. The four-point bending device (Miracle Technology Co., Ltd., Chengdu, China) is divided into three parts: Mechanical power systems, host computer and strain-loading dish. The deformation displacement, loading frequency and loading time were set via the host computer. An engine was used to generate a mechanical force, which was used to exert a strain onto a petri-dish containing cells via a stamping motion. Once the petri dish was bent, a corresponding force was exerted on the cells in the petri dish.

Using the four-point bending system for mechanical loading, the fibroblasts in the exponential growth phase at passage 4-8 derived from 10 patients were subjected to the loading strain. Parameters were set to a frequency of $0.1 \mathrm{~Hz}$ over $4 \mathrm{~h}$, and cells were subjected to strains of $1,333 \mu(1 \mathrm{~mm})$, $2,666 \mu(2 \mathrm{~mm})$ and 5,333 $\mu(4 \mathrm{~mm})$. The control group samples $(0 \mathrm{~mm})$ were incubated at $37^{\circ} \mathrm{C}$ without any treatment under the same culture conditions.

Detection of ROS. The ability of mechanical stress treatment to increase ROS production in HPLFs was measured by the fluorescent probe 2',7'-dichlorodihydrofluorescein diacetate $\left(\mathrm{H}_{2} \mathrm{DCF}-\mathrm{DA}\right.$; Beyotime Institute of Biotechnology, Shanghai, China). Fibroblasts were subjected to strains of $0,1,333,2,666$ and 5,333 $\mu$ for $4 \mathrm{~h}$ using the four-point bending device. The cells were then washed three times with PBS and incubated for $40 \mathrm{~min}$ at $37^{\circ} \mathrm{C}$ with $1.5 \mu \mathrm{l} \mathrm{H}_{2} \mathrm{DCF}-\mathrm{DA}$ in serum-free medium. Cells were again washed three times with PBS, and fresh serum-free DMEM was added. Images of cells with ROS-associated fluorescence were captured using an inverted fluorescence microscope (CKX31; Olympus, Tokyo, Japan), and images were analyzed using Image J version 1.46 software (National Institutes of Health, Bethesda, MD, USA).

JC-1 staining. JC-1 (Beyotime Institute of Biotechnology) is a sensitive fluorescent probe which can be utilized to measure the $\Delta \Psi \mathrm{m}$. In healthy cells with high mitochondrial $\Delta \Psi \mathrm{m}, \mathrm{JC}-1$ spontaneously forms complexes known as JC-1 aggregates with intense red fluorescence. By contrast, in apoptotic or unhealthy cells with low $\Delta \Psi \mathrm{m}$, JC-1 remains in the monomeric form, which shows only green fluorescence. After the 


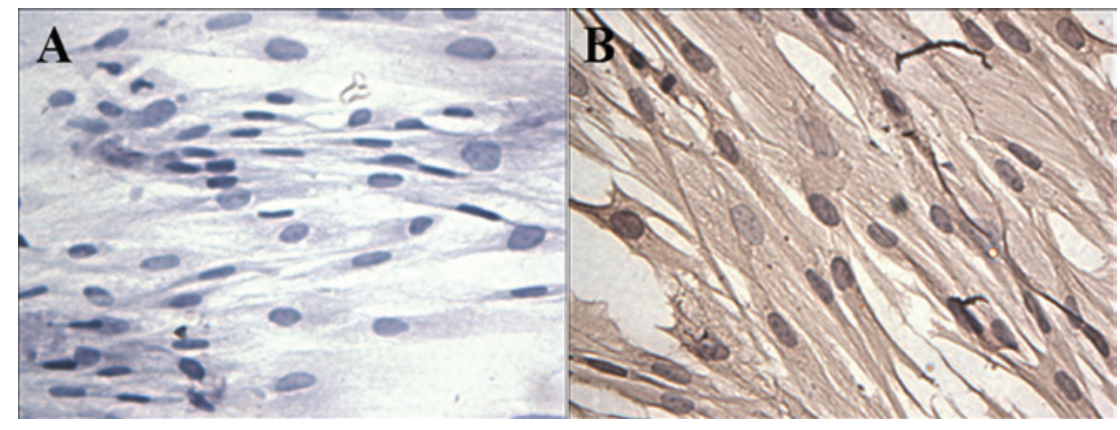

Figure 1. Immunohistochemical staining for (A) cytokeratin 19 and (B) vimentin in cultured parametrial ligament fibroblasts (magnification, $\mathrm{x} 400$ ).

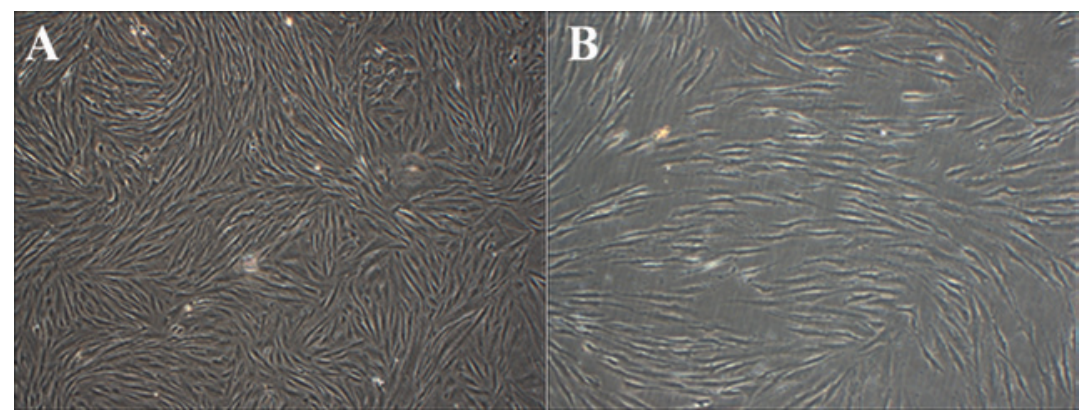

Figure 2. Representative images demonstrating cell morphological changes of human parametrial ligament fibroblasts. Cell morphology (A) prior to stretching and (B) following mechanical stretching by application of a $1,333-\mu$ strain for $4 \mathrm{~h}$ (magnification, $\mathrm{x} 40$ ).

fibroblasts had been subjected to the respective strains for $4 \mathrm{~h}$, they were rinsed twice with PBS and incubated with a mixture of $2 \mu \mathrm{l}$ JC-1 staining solution and $2 \mathrm{ml}$ serum-free medium in the dark at $37^{\circ} \mathrm{C}$ for $30 \mathrm{~min}$ according to the manufacturer's instructions. The cells were then washed with JC-1 staining buffer twice, and $2 \mathrm{ml}$ serum-free DMEM was added to each specimen. The JC-1 fluorescence was observed under the inverted fluorescence microscope. Image $\mathbf{J}$ software was employed to analyze the red and green fluorescence intensity. The ratio of red to green fluorescence was calculated, which was indicative of the $\Delta \Psi \mathrm{m}$.

Detection of apoptosis by Annexin V/propidium iodide $(P I)$. The HPLFs were subjected to strains as mentioned above. Subsequently, the apoptotic rate was determined by Annexin V/PI (Beyotime Institute of Biotechnology) double staining according to the manufacturer's instructions. Briefly, cells from different groups were harvested, washed with ice-cold PBS twice and re-suspended in $400 \mu \mathrm{l}$ binding buffer. $5 \mu \mathrm{l}$ fluorescein isothiocyanate-conjugated Annexin $\mathrm{V}$ and $10 \mu \mathrm{l} \mathrm{PI}$ were added, followed by incubation for $20 \mathrm{~min}$ in the dark at room temperature. The apoptotic rate was analyzed by flow cytometry (BD LSR II; BD Biosciences, Franklin Lakes, NJ, USA) using Flow Jo software 7.6 (BD Biosciences). The cells which stained positive for Annexin $\mathrm{V}$ and negative for PI were considered to be early apoptotic, while those which were positive for both were identified as late apoptotic cells. The apoptotic rates were expressed as the percentage of the total cell population.

Statistical analysis. Statistical analyses were performed using SPSS 16.0 (SPSS Inc., Chicago, IL USA). Image J software-generated fluorescence values and cell ratios in Flow Jo software 7.6 format were imported to SPSS, and the significance of differences between groups was tested by analysis of variance. $\mathrm{P}<0.05$ was considered to indicate a statistically significant difference between values.

\section{Results}

Primary culture and identification of HPLFs. The fibroblasts mainly appeared as long spindles, but also as irregular triangles, polygons and other shapes as observed by light microscopy (Fig. 1). Cells were connected to each other to form a network structure. Immunohistochemical staining showed that cells were negative for cytokeratin (Fig 1A) and positive for vimentin (Fig. 1B).

Mechanical stress loading leads to morphological changes of HPLFs. Under all mechanical stress loading conditions, cell morphology was atrophied, cell junctions appeared loose and weakened. Sections of the fibroblasts suffered a deformation from long spindles to round bodies. A 4-mm strain increased the rate of cell disruption and cell shedding (Fig. 2).

Mechanical stress loading increases intracellular ROS levels in HPLFs. In the control group, the ROS-associated fluorescence was weak (Fig. 3A and B), while in the experimental groups, ROS fluorescence was enhanced after mechanical stress loading (Fig 3C-H). The quantified fluorescence intensity following exposure to strains of $0,1,333,2,666$ and 5,333 $\mu$ was $10.27 \pm 1.53,20.13 \pm 4.55,23.55 \pm 6.99$ and $64.15 \pm 12.68$, respectively, indicating that ROS production was significantly increased in the experimental groups compared with that in the control group (Fig. 3I). 

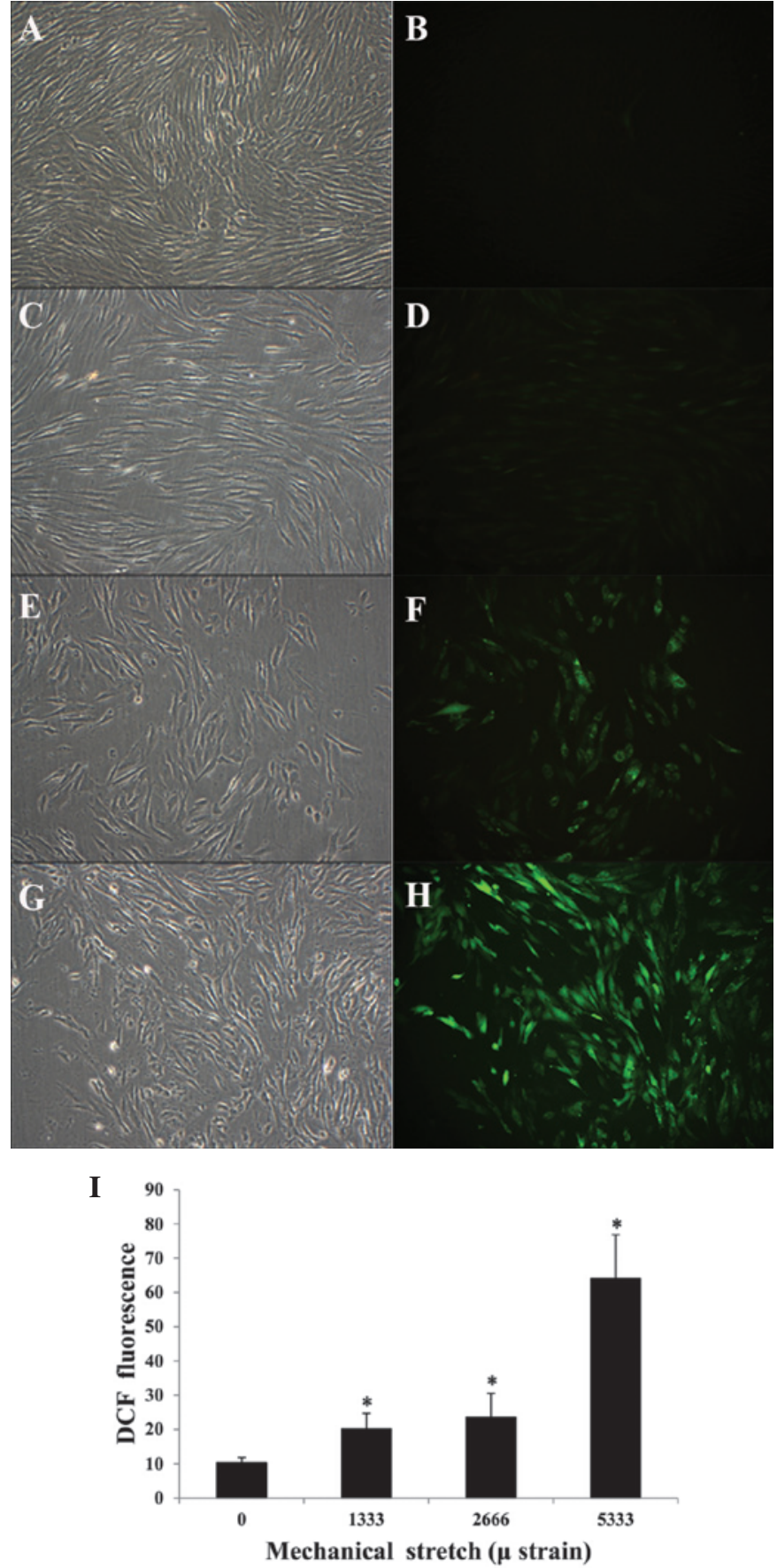

Figure 3. (A) Cells were subjected to various degrees of mechanical strain for $4 \mathrm{~h}$ and stained with DCF-DA (green) to detect ROS. (A and B), control group; (C and D) 1333- $\mu$ strain; (E and F) 2666- $\mu$ strain; (G and H) 5333- $\mu$ strain (magnification, x100). Transmitted light images are shown on the left and fluorescence microscopy images are shown on the right. (I) The intensity of DCF-mediated fluorescence was quantified using Image J software and reflected the levels of intracellular ROS. ROS production was increased after mechanical strain and mechanical stress induced the production of ROS in a loading-dependent pattern. Values are expressed as the mean fluorescence intensity \pm standard deviation of five fields of view per group (magnification, $\mathrm{x} 100) .{ }^{*} \mathrm{P}<0.01$ vs. control. ROS, reactive oxygen species; DCF-DA, 2',7'-dichlorodihydrofluorescein diacetate.

Mechanical stress loading decreases the $\triangle \Psi m$ in HPLFs. In the control cells, the JC-1 dye emitted a red fluorescence, indicating an intact $\Delta \Psi \mathrm{m}$ (Fig. 4A-C). After mechanical stress loading, the red fluorescence of the JC-1 dye was weakened, and green fluorescence was enhanced (Fig. 4D-I). Quantitative
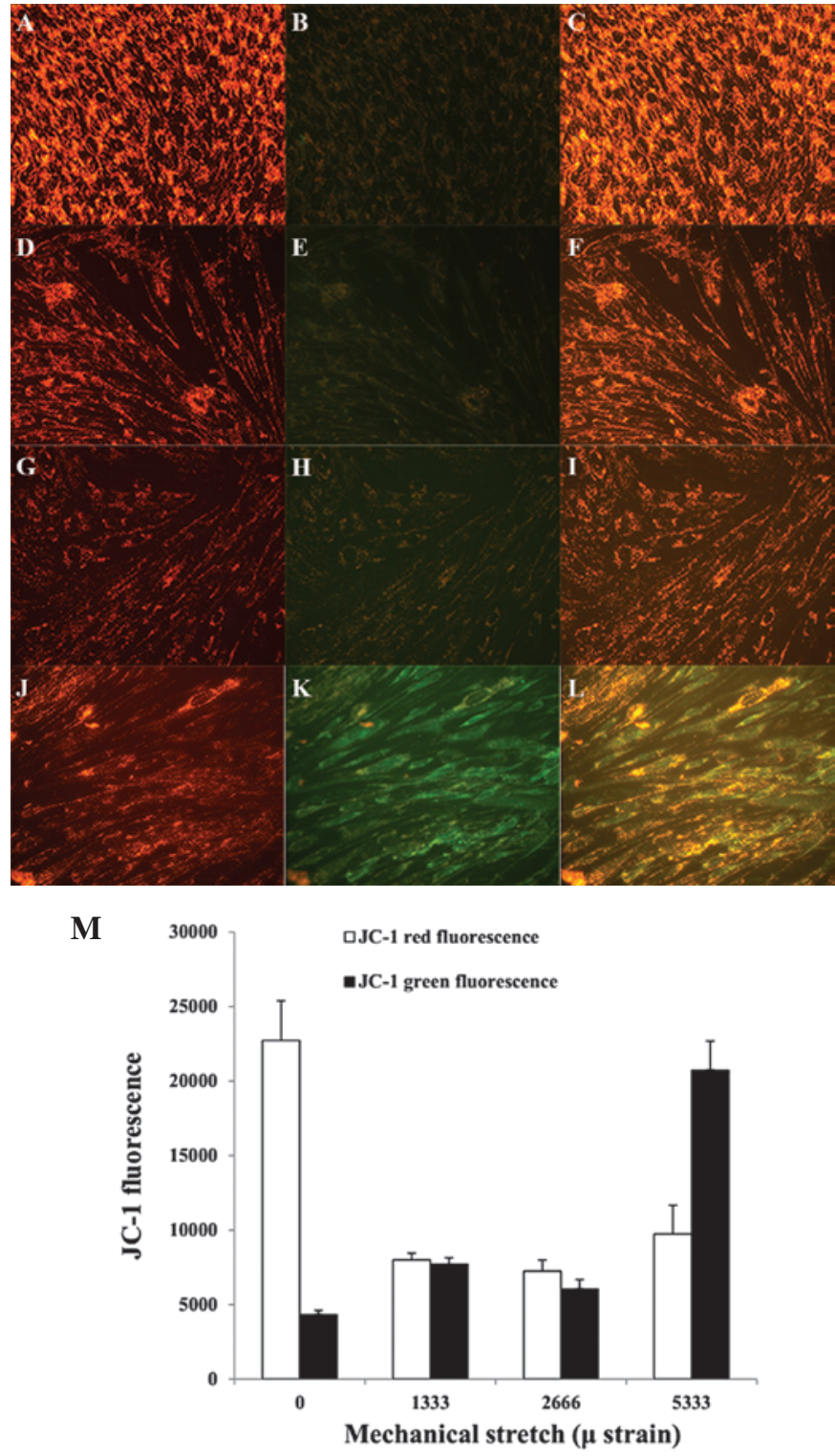

Ratio(red/green) $5.26 \pm 0.621 .03 \pm 0.14^{*} \quad 1.19 \pm 0.03^{*} \quad 0.44 \pm 0.01^{*}$

Figure 4. Assessment of the mitochondrial membrane potential by JC-1 staining. Representative fluorescence photomicrographs of in-situ JC-1-stained cells in various treatment groups (magnification, x200). (A-C), Control; (D-F), 1,333- $\mu$ strain; (G-I), 2,666- $\mu$ strain; (J-L), 5,333- $\mu$ strain. Images on the left show red fluorescence, indicating a high mitochondrial membrane potential; the middle panel shows green fluorescence, indicative of a loss of mitochondrial membrane potential. The right-hand panel shows merged red and green fluorescence. (M) Red and green fluorescence intensity was quantified by Image $\mathbf{J}$ software. The ratio of red to green fluorescence intensity is indicative of the mitochondrial membrane potential. Values are expressed as the mean fluorescence intensity \pm standard deviation of five fields of view per group.

analysis of the fluorescent microscopy images with Image $\mathbf{J}$ software showed that the ratio of red to green fluorescence of cells subjected to strains of $0,1,333,2,666$ and $5,333 \mu$ was $5.26 \pm 0.62,1.03 \pm 0.014,1.19 \pm 0.03$ and $0.44 \pm 0.01$, respectively (Fig. 4M). The decreased red to green fluorescence ratio of JC-1 confirmed the decline in the $\Delta \Psi \mathrm{m}$.

Mechanical stress loading increases the apoptotic rate of HPLFs. Apoptosis was assessed using Annexin V/PI double-staining and flow cytometric analysis (Fig. 5A). The 


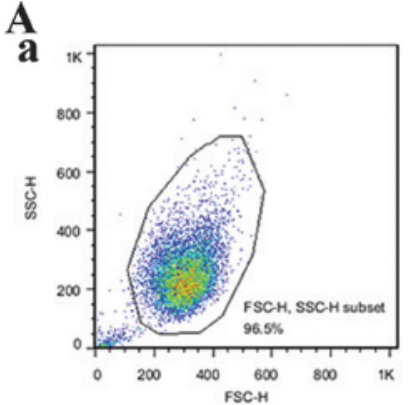

c
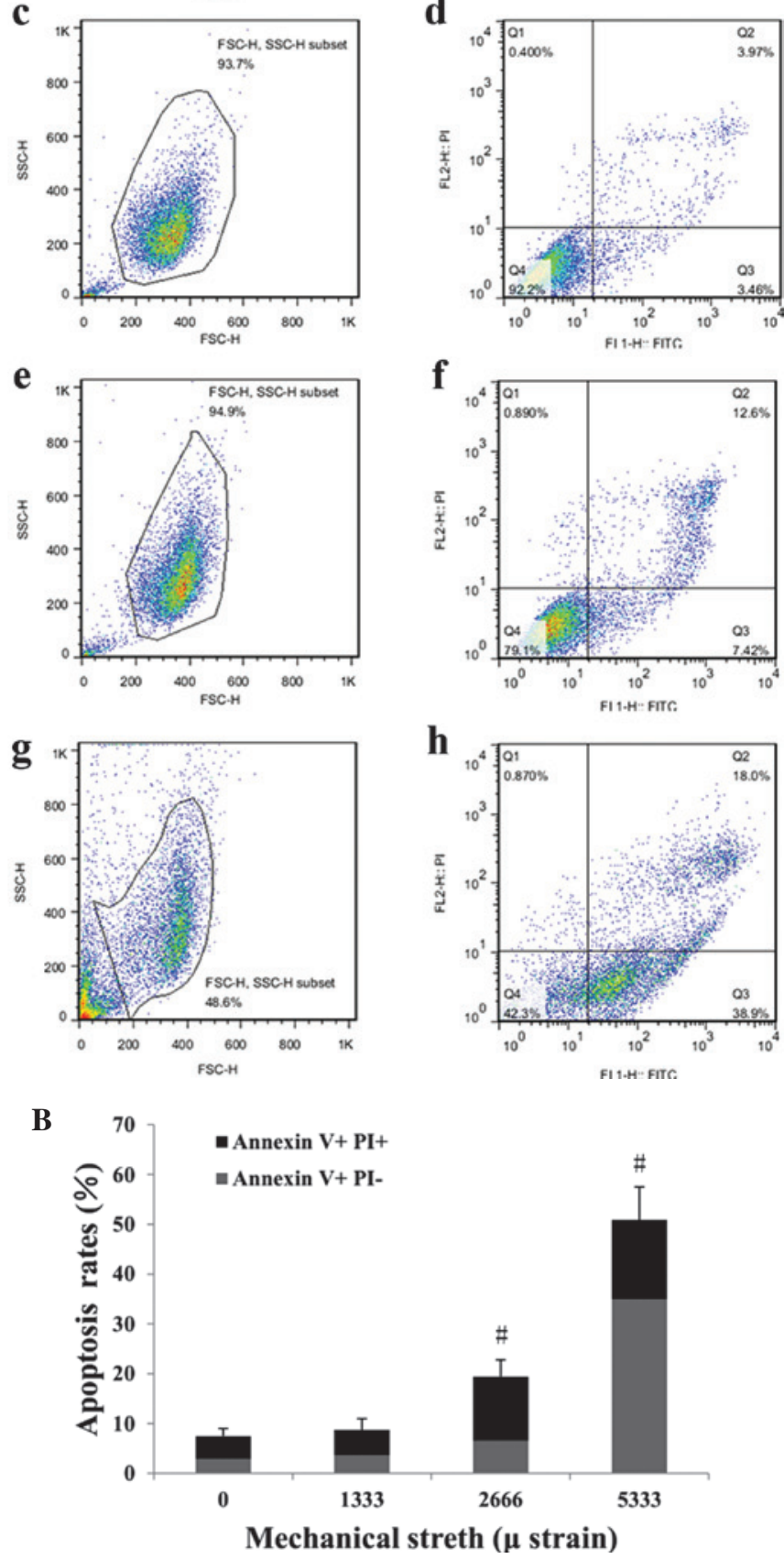

Figure 5. Mechanical stress-induced apoptosis of human parametrial ligament fibroblasts occurs in a loading-dependent manner. (A) Representative dot plots of cell apoptosis by flow cytometry analysis after Annexin V/PI dual staining. The apoptotic rate was determined as the percentage of Annexin V-positive cells, with early apoptotic cells being PI-negative and late apoptotic cells being PI-positive. (a and b) control; (c and d) 1,333- $\mu$ strain; (e and f) 2,666- $\mu$ strain; (g and h) 5,333- $\mu$ strain. (B) Quantified apoptotic rates of human parametrial ligament fibroblasts treated with various intensities of mechanical strains for $4 \mathrm{~h}$. Values are expressed as the mean \pm standard deviation of three independent experiments. ${ }^{*} \mathrm{P}<0.05 ;{ }^{*} \mathrm{P}<0.01$ vs. control. PI, propidium iodide FITC, fluorescein isothiocyanate; FSC, forward scatter; SSC, side scatter. quantified results showed that a strain of $1,333 \mu$ did not markedly affect the apoptotic rate, while strains of 2,666 and $5,333 \mu$ significantly enhanced the apoptotic rate compared with that of the control group (Fig. 5B). In particular, a strain of 5,333 $\mu$ had a marked apoptotic effect, leading to an apoptotic rate of $\sim 50 \%$ among non-necrotic cells $(\sim 50 \%$ of total population) with a large amount of cell debris (Fig. 5Ag and h).

\section{Discussion}

Over a woman's lifetime, her pelvic floor tissues bear various mechanical stresses, including pregnancy, childbirth, defecation, cough and normal gravity, which contribute to an increased intra-abdominal pressure (13). POP is thought to be caused by a decrease in the biomechanical properties of pelvic supports (14). Uterine cardinal ligaments and utero-sacral ligaments surrounding the cervix are the major ligaments to bear pressures and maintain the uterus' normal position, and patients with POP who suffer an uterine prolapse attack present with a decreased strength of these ligaments (15). The decreasing biomechanical properties are linked with tissue components, including cells and extracellular matrix (ECM); the ECM is synthesized and secreted by fibroblasts in response to strain. Therefore, the present study focused on the mechanisms of the effects of mechanical strain in the pathogenesis of POP. A four-point bending device was used to exert cyclic tensions on fibroblasts of uterus ligaments in order to mimic the intra-abdominal mechanical strain. The present study aimed at exploring whether the cytological changes of POP, including oxidative stress injury, mitochondrial apoptosis and cellular apoptosis, matched with the mechanical strain loading on the fibroblasts of uterus ligaments. It has been reported that four-hour mechanical strain loading on fibroblasts of periodontal ligaments by a four-point bending device led to obvious changes of these cells (11). The experimental conditions of this previous study, combined with the results of previous preliminary experiments by our group, were used to select the strains exerted on the uterine ligamental fibroblasts in the present study $(1,333,2,666$ or $5,333-\mu$ strains over four hours).

In the present study, morphological observation indicated that part of the fibroblasts suffered a deformation from long spindles to round bodies under the action of mechanical force. The cells became easily detached from the petri-dishes, the cell morphology was atrophied and cell junctions appeared loose, which was similar to the observations of a previous study (16). It has been reported that the morphology of fibroblasts undergoes regular changes, and that cells are oriented perpendicular to the direction of the strain (17). The present study failed to observe any obvious changes in cell orientation, which may be due to the short time of mechanical stress loading. A certain amount of time is required for the cell orientation to change. Future studies by our group will further investigate this matter.

Furthermore, the production of ROS was assessed in HPLFs subjected to mechanical stress. ROS-associated fluorescence in the HPLFs increased with the enhancement of mechanical stress loading, particularly under the mechanical strain of $5333 \mu$. ROS are known to be the main source of oxidative stress, which may cause oxidative modifications of DNA, lipids and proteins. Under physiological conditions, modest ROS have positive effects on cells by scavenging endotoxin and are involved in the regulation of cell growth, serving as a second 
messenger (18). However, when the production of ROS becomes excessive, it may have harmful effects on cells. Similarly, human pelvic supportive structures continuously inherit physiological mechanical stress under normal circumstances, which may also have a beneficial influence on cells. Numerous studies have shown that mechanical forces can lead to oxidative damage in variety of non-pelvic floor cell types $(5-6,19)$. Therefore, the present study hypothesized that these risk factors, including pregnancy, childbirth, chronic cough and constipation, increase the abdominal pressure, which exerts excessive mechanical forces and may induce ROS production, leading to imbalances in the redox equilibrium of pelvic organs. The results of the present study demonstrated that low mechanical strain induced a slight increase in ROS, which was further elevated with the enhancement of mechanical stress.

The present study assessed the effects of mechanical stress on the $\Delta \Psi \mathrm{m}$ of HPLFs. Mitochondria are among the primary organelles targeted by oxidative stress, as excessive ROS can directly harm mitochondria (20). Low permeability and the electrochemical proton gradient of the mitochondrial inner membrane are the basis for maintaining the $\Delta \Psi \mathrm{m}$ and essential for normal physiological function. The $\Delta \Psi \mathrm{m}$ decreases in the early stage of apoptosis, triggering a series of biochemical changes, including the release of cytochrome $\mathrm{C}$ and $\mathrm{B}$-cell lymphoma 2 (Bcl-2) as well as the activation of caspase. Cytochrome $\mathrm{C}$ and $\mathrm{Bcl}-2$, as caspase activators, regulate energy metabolism and cellular apoptosis. Caspases can trigger the cascade reaction of cellular apoptosis, resulting in cellular death. It has been reported that mechanical strain can cause a decrease of myocardial $\Delta \Psi \mathrm{m}(21,22)$. The results of the present study indicated that the $\Delta \Psi \mathrm{m}$ of HPLFs decreased following mechanical stress. When HPLFs were subjected to strains of 1,333 and $2,666 \mu$, the $\Delta \Psi \mathrm{m}$ dropped as indicated by a ratio of red to green fluorescence of $\sim 1$ as opposed to $\sim 5$ in the control group. Of note, a mechanical strain of 5,333 $\mu$ caused a distinct increase of green fluorescence intensity and the ratio of red to green fluorescence intensity was $<0.4$. These results proved that mechanical stress decreased the $\Delta \Psi \mathrm{m}$. The decline of the $\Delta \Psi \mathrm{m}$ can active the mitochondrial apoptotic pathway, which results in cellular apoptosis.

Mechanical stress, such as tension and fluid shear stress, and the cellular apoptotic rate are linked, and when cells are exposed to forces above physical stimulation, the apoptotic rate increases in proportion with the magnitude of the force $(23,24)$. However, to date, the underlying mechanism of mechanical stress-induced cellular apoptosis has remained elusive. Li et al (25) suggested that mechanical load-induced apoptosis of articular cartilage may be associated with the release of a large amount of calcium ions by the endoplasmic reticulum, leading to the activation of a mitochondrial apoptotic pathway. Ding et al (26) reported that compression-induced apoptosis of nucleus pulposus cells was associated with ROS-mediated mitochondrial apoptosis. A histological study of tissues from patients with POP also proved that the mitochondrial apoptotic pathway was activated (10). Thus, the present study hypothesized that mechanical stress may cause apoptosis of fibroblasts from uterus ligaments through the activation of the mitochondrial apoptotic pathway. The present study showed that a mechanical strains significantly increased the cellular apoptosis rate to $>17$ times that of the control cells. Furthermore, the present study and our preliminary results indicated that high mechanical strains led to increased cellular detachment from the slide, significant increases in intracellular ROS production, decreases in the $\Delta \Psi \mathrm{m}$ and a high level of apoptosis, which proceeded via the mitochondrial pathway. The results of the present study verified its preliminary hypothesis that mechanical stress initiated mitochondrial-depended apoptosis in HPLFs via injuring their mitochondria on the account of oxidative stress. Furthermore, cellular fragmentation was enhanced by a strain of 5,333 $\mu$, leading of a population of non-necrotic cells of only $50 \%$ out of which $50 \%$ were apoptotic, while a strain of 2,666 $\mu$ resulted in $95 \%$ non-necrotic cells. The low amount of necrosis in the lower-strain groups may be due to the short time (four hours) over which the strain was applied, and in which time cellular apoptosis mainly stayed in the early stage. It is possible that the increased amount of cellular fragments was caused by excessive mechanical stress, which directly damaged cellular integrity. Therefore, the strain of 5,333 $\mu$ mechanically damaged the fibroblasts of uterus ligaments and the oxidative stress-induced mitochondrial pathway may therefore not be the only mechanism leading to cellular apoptosis under high mechanical stress loading. These remaining questions will be addressed in further studies by our group.

As one of the most important risk factors involved in POP pathogenesis, mechanical strain has already drawn the attention of numerous researchers. Mechanical indicators of POP patients' pelvic supports and possible intervention methods have been studied previously (27). At present, there is no established animal model or fibroblasts cell line of uterus ligaments to be experimented on, which has restricted the development of POP research. The present study clarified the pathogenesis of POP from the angle that mechanical strain induced oxidative stress, which in turn caused apoptosis of HPLFs via a mitochondrial pathway, and provided a theory for the association between oxidative stress-associated cell injury and POP pathogenesis. However, the present study only provided preliminary information on the effects of mechanical strain on the development of POP, which require further in-depth mechanistic study. In particular, the effects of various intensities and periods of mechanical strain require to be tested. Further study is also required regarding the cytomechanical differences between HPLFs from POP and non-POP patients, and the possible attenuating effects of anti-oxidative agents on the development of POP may be worth investigating.

\section{Acknowledgements}

This work was supported by the National Natural Science Foundation of China (no. 81270684) and the Foundation of Collaborative and Innovation Projects of Wuhan University School of Medicine (no. 523-266078).

\section{References}

1. Peng P, Zhu L, Lang J, Wang WY and Shi HH: Unilateral sacrospinous ligament fixation for treatment of genital prolapse. Chin Med J (Engl) 123: 1995, 2010.

2. Jelovsek JE, Maher C and Barber MD: Pelvic organ prolapse. Lancet 369: 1027-1038, 2007.

3. Wondrak GT, Roberts MJ, Jacobson MK and Jacobson EL: 3-hydroxypyridine chromophores are endogenous sensitizers of photooxidative stress in human skin cells. J Biol Chem 279: 30009-30020, 2004 
4. Mizutari K, Ono T, Ikeda K, Kayashima K and Horiuchi S: Photo-enhanced modification of human skin elastin in actinic elastosis by N(epsilon)-(carboxymethyl) lysine, one of the glycoxidation products of the maillard reaction. J Invest Dermatol 108: 797-802, 1997.

5. Lu D and Kassab GS: Role of shear stress and stretch in vascular mechanobiology. J Roy Soc Interface 8: 1379-1385, 2011.

6. Tan JL: Cell proliferation, differentiation, apoptosis under a serial stretches and the underlying mechanisms. PhD dissertation. The Fourth Military Medical University, Xi'an, 2010.

7. Choy KW, Liu YM, Chu CY, Wang CC, Lui WT, Lee LL, Pang MW, Rogers MS and Yip SK: High isoprostane level in cardinal ligament-derived fibroblasts and urine sample of women with uterine prolapse. BJOG 115: 1179-1183, 2008

8. Visco AG and Yuan L: Differential gene expression in pubococcygeus muscle from patients with pelvic organ prolapse. Am J Obstet Gynecol 189: 102-112, 2003.

9. Makpol S, Azura Jam F, Anum Mohd Yusof Y and Zurinah Wan Ngah W: Modulation of collagen synthesis and its gene expression in human skin fibroblasts by tocotrienol-rich fraction. Arch Med Sci 7: 889-895, 2011.

10. Kim E J, Chung N, Park SH, Lee KH, Kim SW, Kim JY, Bai SW and Jeon MJ: Involvement of oxidative stress and mitochondrial apoptosis in the pathogenesis of pelvic organ prolapse. J Urol 189: 588-594, 2013.

11. Wen Y, Ho JY, Polan ML and Chen B: Expression of apoptotic factors in vaginal tissues from women with urogenital prolapse. Neurourol and Urodynam 30: 1627-1632, 2011.

12. Bao R, Liu X and Zhou J: Primary culture and identification of human fibroblasts. J XinX Med Coll 5: 015, 2011 (In Chinese)

13. Chow D and Rodríguez LV: Epidemiology and prevalence of pelvic organ prolapse. Curr Opin Urol 23: 293-298, 2013.

14. Jean-Charles C, Rubod C, Brieu M, Boukerrou M, Fasel J and Cosson M: Biomechanical properties of prolapsed or non-prolapsed vaginal tissue: impact on genital prolapse surgery. Int Urogynecol J 21: 1535-1538, 2010.

15. Lang JH and Zhang XD: Obstetrics and Gynecology Clinical Anatomy. 2010 edition. Shandong Science and Technology Press, Jinan, pp69, 2010

16. Ewies AA, Elshafie M, Li J, Stanley A, Thompson J, Styles J, White I and Al-Azzawi F: Changes in transcription profile and cytoskeleton morphology in pelvic ligament fibroblasts in response to stretch: The effects of estradiol and levormeloxifene. Mol Human Reprod 14: 127-135, 2008.
17. Li XN: Study on the methods and platform of image processing for cellular biomechanical experiments. PhD dissertation. Sichuan University, Chengdu, 2003.

18. Fransen M, Nordgren M, Wang B and Apanasets O: Role of peroxisomes in ROS/RNS-metabolism: Implications for human disease. Biochim Biophys Acta 1822: 1363-1373, 2012.

19. Grote K, Flach I, Luchtefeld M, Akin E, Holland SM, Drexler H and Schieffer B: Mechanical stretch enhances mRNA expression and proenzyme release of matrix metalloproteinase-2 (MMP-2) via NAD $(\mathrm{P}) \mathrm{H}$ oxidase-derived reactive oxygen species. Circ Res 92: e80-e86, 2003.

20. Sinha K, Das J, Pal PB and Sil PC: Oxidative stress: The mitochondria-dependent and mitochondria-independent pathways of apoptosis. Arch Toxicol 87: 1157-1180, 2013.

21. Teshima Y, Takahashi N, Thuc LC, Nishio S, Nagano-Torigoe Y, Miyazaki H, Ezaki K, Yufu K, Hara M, Nakagawa M and Saikawa T: High-glucose condition reduces cardioprotective effects of insulin against mechanical stress-induced cell injury. Life Sci 87: 154-161, 2010.

22. Choudhary R, Baker KM and Pan J: All-trans retinoic acid prevents angiotensin II- and mechanical stretch-induced reactive oxygen species generation and cardiomyocyte apoptosis. J Cell Physiol 215: 172-181, 2008

23. Meyer T, Meyer U, Stratmann U, Wiesmann HP and Joos U: Identification of apoptotic cell death in distraction osteogenesis. Cell Biol Int 23: 439-446, 1999.

24. Ning QM and Qang XR: Early apoptosis induced by mechanical stretch in human alveolar typeIIepithelial cells. Journal of Shanghai Jiao Tong University (Medical Science Edition) 27: 961-964, 2007

25. Li H, Zhang XY, Wu TJ, Cheng W, Liu X, Jiang TT, Wen J, Li J, Ma QL and Hua ZC: Endoplasmic reticulum stress regulates rat mandibular cartilage thinning under compressive mechanical stress. J Biol Chem 288: 18172-18183, 2013.

26. Ding F, Shao ZW, Yang SH, Wu Q, Gao F and Xiong LM: Role of mitochondrial pathway in compression-induced apoptosis of nucleus pulposus cells. Apoptosis 17: 579-590, 2012.

27. Miao YL, Hu CH, Wang JL, et al: The preliminary study on biomechanical properties of posterior vaginal wall tissue of the patient with rectocele. Chinese J Clin Obstet Gynecol 9: 180-183, 2008 (In Chinese). 